\title{
Reinforcement of a reinforcing behavior: Effect of sucrose concentration on wheel-running rate
}

Terry W.Belke1, W. David Pierce², Alexandra C. Fisher ${ }^{1}$, and Mandy R. Lecocq ${ }^{3}$

${ }^{1}$ Mount Allison University, Sackville, New Brunswick

${ }^{2}$ University of Alberta, Edmonton, Alberta

${ }^{3}$ Concordia University, Montreal, Quebec

Corresponding author:

Terry W. Belke

Department of Psychology

Mount Allison University

Sackville, New Brunswick

Canada E4L 1C7

5063642459

tbelke@mta.ca

Running Head: REINFORCEMENT OF OPERANT WHEEL RUNNING 


\begin{abstract}
Wheel running, unlike typical operant behavior, generates its own automatic reinforcement that alters the control exerted by extrinsic reinforcement on wheel running. The current study investigated the implications of the automatic reinforcement of wheel running by arranging different sucrose concentrations as extrinsic reinforcement for operant wheel running in ad-lib fed and food-deprived rats. Eleven female Long Evans rats ran on fixed revolution 30 schedules that delivered a drop of sucrose solution as reinforcement. Sucrose concentration varied across values of $0 \%, 2.5 \%, 5 \%, 10 \%$, and $15 \%$ sucrose $(\mathrm{w} / \mathrm{v})$. Results showed that under ad-lib feeding, only the highest concentrations increased operant wheelrunning rate. By contrast, under deprivation, all concentrations of sucrose increased the rate of wheel running. Despite the differences in sucrose-reinforced operant wheel-running rates by deprivation level (ad lib vs. deprived), wheel-running rates did not differ at the highest concentrations. Prior research on operant lever pressing, a response generating low (or no) automatic reinforcement, has shown considerably higher lever-pressing rates as a function of increasing amounts of sucrose reinforcement when rats are food deprived. Together, these previous observations and the current study suggest that automatic reinforcement generated by an operant decreases the control exerted by extrinsic reinforcement. Additionally, the regulation by extrinsic reinforcement on automatically reinforcing behavior depends on the organism's motivation or deprivation level (ad lib vs. deprived).
\end{abstract}


Keywords: operant wheel-running rate; automatic reinforcement; extrinsic reinforcement; sucrose concentration; revolution; rat

\section{Introduction}

Running in a wheel is behavior that can function as reinforcement for an operant behavior (e.g., Belke, 1997; Belke \& Pierce, 2014, 2015; Collier \& Hirsch, 1971; Iversen, 1993; Kagan \& Berkun, 1954; Premack, 1962; Premack, Schaeffer, \& Hundt, 1964) or as an operant behavior that produces reinforcement (Belke, Mann, \& Pierce, 2015; Belke \& Pierce, 2014, 2015; Iso, 1996; Skinner \& Morse, 1958). Operant wheel running generates its own the automatic reinforcement that maintains this behavior at a higher operant level than more typical responses such as lever pressing or nose poking (Belke, Mann, \& Pierce, 2015). One implication of the high operant level of wheel running is that extrinsic sucrose reinforcement would show weak response-strengthening effects, especially in free-feeding rats. The objective of the current study was to investigate how different concentrations ( $0 \%$ to $15 \%$ solutions) of sucrose reinforcement controlled operant wheel-running rate in food deprived and non-deprived rats.

Sucrose, or the opportunity to consume sucrose, functions as reinforcement for operant behavior. Previous research (Bailey, Hsiao, \& King, 1985; Belke \& Hancock, 2003; Guttman, 1953; Leslie, 1977; Vigorito, Kruse, \& Carretta, 1994) with rats has shown that lever-pressing rate increases as sucrose concentration increases up to a point and then either levels off or decreases with further increases in concentration, depending on factors such as the schedule, effort, volume, and/or satiation. For example, Bailey, Hsiao, and King assessed the effect of $0.025 \mathrm{M}(.08 \%)$ 
to $1.1 \mathrm{M}(37.7 \%)$ sucrose solutions on lever-pressing rate by Sprague-Dawley rats on a continuous reinforcement (CRF) schedule. With the lever force set at $30 \mathrm{~g}$, lever-pressing rate increased with molarity up to $.5 \mathrm{M}(17.1 \%)$ and then leveled off. Guttman (1953) assessed the effect of sucrose concentrations of 4\%, 8\%, 16\% and $32 \%$ on lever-pressing rates by albino rats on CRF and fixed interval (FI) 1-min reinforcement schedules. On the CRF schedule, lever-pressing rate increased from 4 to $8 \%$, remained unchanged from 8 to $16 \%$, then decreased at $16 \%$. On the FI schedule, lever-pressing rates increased with concentration. Thus, the function relating concentration of sucrose reinforcement to rate of lever pressing depended on the schedule (CRF or FI). In Belke and Hancock (2003), male Wistar rats lever pressed on FI schedules that produced either a drop of sucrose or the opportunity to run for $15 \mathrm{~s}$. With sucrose concentration manipulated across concentrations of $0 \%$, $2.5 \%, 5 \%, 10 \%$, and $15 \%$, lever-pressing rates increased from $0 \%$ to $10 \%$ but remained unchanged at 15\%, a finding inconsistent with the increasing function reported by Guttman (1953).

Skinner and Morse (1958) were the first to investigate wheel running as operant behavior on a FI 5-min schedule with food pellets delivered as reinforcement. Results for two food-deprived brown rats showed higher rates of wheel running with food reinforcement; also, rats developed a break-and-run pattern of responding characterized by a postreinforcement pause (PRP) followed by a high rate of wheel running that decelerated near the end of the reinforcement interval. Subsequently, Iso (1996) compared wheel-running rates in rats receiving food reinforcement for running on fixed ratio (FR) 40 or FI 60-s schedules with 
yoked rats receiving food independent of wheel running. Running rates did not differ between the yoked and reinforcement groups; however, rats receiving food reinforcement showed response patterns consistent with the reinforcement schedules.

More recently, Belke and Pierce (2015) investigated the effect of sucrose solution as reinforcement for wheel-running rate on a multiple schedule. In this study, wheel running served as reinforcement for lever pressing in one component and as operant behavior producing sucrose solution in the other component. Results showed that wheel-running rates that produced $15 \%$ sucrose (operant running component) were higher than wheel-running rates that functioned as reinforcement for lever pressing (reinforcement component) — confirming a reinforcement effect of sucrose. When $15 \%$ sucrose solution was replaced with water ( $0 \%$ sucrose), operant wheel-running rates decreased by approximately $26 \%$ from 61.25 to 44.62 revolutions/min, on average. Notably, wheel running still occurred at a high rate during extinction.

Using the same multiple-schedule procedure, Belke, Mann, and Pierce (2015) showed that operant lever-pressing rate declined by $90 \%$ from 229.0 presses/min to 19.3 presses/min when $15 \%$ sucrose was replaced with water ( $0 \%$ sucrose). But, operant wheel-running rate only decreased by $24 \%$ from 58.8 to 44.1 revolutions/min. The relatively limited decrease in wheel-running rate with the removal of sucrose occurs because wheel running, unlike lever pressing, generates its own automatic reinforcement. As a result, wheel running is maintained at a higher rate in extinction, and at a higher operant level, than lever pressing. With 
respect to the current study, the automatic reinforcement generated by wheel running limits the range of wheel-running rates controlled by different concentrations of sucrose reinforcement, especially when rats are not food deprived.

In terms of food deprivation, it is notable that the value of sucrose and wheel running as reinforcing consequences increase with deprivation (Belke, 1996, 2004; Belke \& Pierce, 2009, 2016). That is, both the opportunity to run and the opportunity to consume sucrose support higher operant rates when rats are food deprived. At $0 \%$ sucrose, deprived rats should run in wheels at higher rates than non-deprived animals, and wheel-running rates for deprived rats should increase with concentration of sucrose reinforcement. On the other hand, non-deprived rats would run in wheels for automatic reinforcement and extrinsic sucrose reinforcement would have more limited effects on wheel-running rates-generating a slowly increasing rate of wheel running with variation in sucrose concentration or perhaps even a flat function with no changes in wheel-running rate as sucrose concentration increases. The current study was designed to investigate the functional relationship between wheel-running rate and sucrose reinforcement concentration ( $0 \%$ to $15 \%$ solutions) in the same rats when non-deprived and deprived.

\section{Methods}

\subsection{Participants}


The experiment was conducted using 12 female Long Evans rats obtained from Charles River Laboratories in Saint-Constant, Quebec. Rats were housed in pairs in polycarbonate cages $(48.3 \mathrm{~cm}$ x $26.7 \mathrm{~cm} \times 20.3 \mathrm{~cm})$. Heat-treated beta chips and paper towel were used as bedding. Lighting in the colony room was on a 12-h light/dark cycle (lights on at 0730). Rats were fed Prolab R-M-H 3000 lab chow and provided distilled water. Water was freely available within home cages. This research was conducted in accord with the guidelines of the Canadian Council on Animal Care under a protocol approved by the Mount Allison Animal Care Committee.

\subsection{Apparatus}

Two Wahmann and two Lafayette activity wheels (350 $\mathrm{mm}$ in diameter) were used in the present experiment. 24 VDC lights were mounted on the sides of the wheel frame to illuminate the interior of the wheel chamber. Wheel revolutions were recorded by a microswitch attached to the wheel frame. A solenoid was attached to the base of the wheel and when activated, the solenoid caused a rubber tip attached to a metal shaft to contact the outer edge of the wheel to prevent it from turning. The wheels were located in sound-attenuated cubicles equipped with electric fans to circulate air and diminish background noise.

A metal panel (180 mm x $185 \mathrm{~mm} \times 2 \mathrm{~mm}$ ) containing a liquid receptacle and two stimulus lights was attached to the $90 \mathrm{~mm}$ x $70 \mathrm{~mm}$ opening of each wheel. The stimulus lights, positioned $60 \mathrm{~mm}$ from the sides and $50 \mathrm{~mm}$ from the top of the metal panel, were not used in the current study. The liquid receptacle $(60 \mathrm{~mm}$ x 30 $\mathrm{mm} \times 35 \mathrm{~mm}$ ) was situated $160 \mathrm{~mm}$ from each side of the panel and $40 \mathrm{~mm}$ from the 
base of the panel to the base of the liquid receptacle. Cylindrical dispensers attached to a solenoid valve were held in place above the liquid receptacle. Experimental events were controlled and the data recorded using Borland Turbo Pascal 4.0 programs run on PC computers interfaced to the wheels through parallel ports.

\subsection{Procedure}

\subsubsection{Training}

Initially, rats were placed in the activity wheels that were programmed to turn freely for $30 \mathrm{~min}$ and the number of revolutions that occurred during that period was recorded. Following 28 sessions of free running, rats were placed on a FR 30 schedule. A session commenced with the release of the brake. After 30 revolutions, the brake engaged and the chamber lights were illuminated for $3 \mathrm{~s}$. This 3-s interval was composed of a $0.5 \mathrm{~s}$ activation of the solenoid valve to deliver $.1 \mathrm{ml}$ of solution ( $0 \%$ to $15 \%$ sucrose) followed by a 2.5 -s interval for consumption of that solution. Upon completion of this 3-s consumption interval, the brake released and the chamber lights extinguished. A session terminated following 20 deliveries of solution or 600 revolutions. Rats were not deprived of wheel running during experimental sessions relative to the $30 \mathrm{~min}$ of free access to running wheels. Over the last five sessions of free access, rats completed an average of 712.5 revolutions $($ S.D. $=172.13)$. For experimental sessions, the animals completed the required 600 revolutions in about 21 min on average (Ad-lib M= 22.43 min; Deprived $M=19.75$ min), running at a similar speed to that on the free-access sessions. After the session, rats were weighed and returned to their home cage. 
Rats were randomly assigned to ascending or descending concentration orders. The ascending group (NQ 1, 2, 3, 4, 9, and 19) received concentrations of $0 \%, 2.5 \%, 5 \%, 10 \%$, and $15 \%$ sucrose while the descending group (NQ 10, 11, 12 , 17 , and 18$)$ received the opposite order $(15 \%, 10 \%, 5 \%, 2.5 \%$, and $0 \%)$. Each concentration remained in effect for 20 sessions. Sessions were conducted 7 days a week and began at 0800 every morning.

After completing five concentrations on free food, the rats were food deprived to a body weight of $260+/-10 \mathrm{~g}$ (target range). As the rats were housed in pairs under ad-lib feeding, deprivation to achieve the target range involved placing each rat in a separate feeding cage with an allotment of food for one hour after an experimental session. Following the hour of limited feeding, rats were returned to their home cages. During this limited-feeding phase, rats on the ascending order received the $0 \%$ solution while rats on the descending order received the $15 \%$ sucrose solution. After 45 sessions, when body weights were within the target range, the second part of this study commenced. Now, under deprived conditions, rats assigned to the ascending and descending groups received the same order of sucrose solutions as arranged in the free-feeding phase. All other conditions were the same.

\subsubsection{Dependent measures.}

During each session, the number of revolutions, the number of reinforcements, time spent wheel running and latency to initiate running following termination of the 3-s outcome interval were recorded by the computer for each reinforcement as well as cumulatively for the session. Wheel-running rates, 
expressed as revolutions/min, were calculated by dividing the 600 revolutions by the time taken to complete 600 revolutions. Mean latency to initiate running (time between the end of the 3-s outcome interval and the first wheel turn) was then calculated by dividing the cumulative latency over the entire session by 20 outcomes.

\subsubsection{Data analysis.}

Wheel-running rates and latencies to initiate running for each concentration, averaged over the last five sessions on each concentration, were analyzed using a three way repeated-measures ANOVA with order of concentration (ascending or descending) as a between-subjects factor, and deprivation condition (ad-lib, food deprived) and sucrose concentration (0\% to 15\%) as within-subjects factors. Posthoc pairwise comparisons were conducted using Bonferroni corrections. One rat on the descending order developed a health problem during the study and in accord with the animal use protocol was removed from the study. Data from this rat were not included in the analyses.

\section{Results}

Figure 1 shows mean wheel-running rates as a function of deprivation condition and concentration for individual rats and the group. For most rats, wheelrunning rates were higher under deprived conditions. Under ad-lib feeding, most rats (NQ $1,9,10,12,17$ and 18), showed an increase in wheel-running rate with concentration. Under deprivation, rats tended to show a mix of increasing rates (NQ $2,9,11,18$, and 19) and increasing then decreasing rates (NQ 1, 3, 4, and10) of wheel running with concentration. The three-way repeated measures ANOVA 
showed significant main effects of deprivation condition (ad lib vs. deprived), $F(1,9)$ $=18.55, \mathrm{p}<.01, \eta_{\mathrm{p}}^{2}=.67$, and concentration, $\mathrm{F}(4,36)=22.50, \mathrm{p}<.001, \eta_{\mathrm{p}}{ }^{2}=.70$, as well as a significant deprivation by concentration interaction, $\mathrm{F}(4,36)=3.26, \mathrm{p}<.05$, $\eta_{\mathrm{p}}^{2}=.24$. For the main effect of deprivation condition, a Bonferroni comparison showed that the mean wheel-running rate under deprivation $(M=37.36$ revolutions/min) was significantly higher than that under ad-lib feeding $(\mathrm{M}=33.08$ revolutions/min), $\mathrm{p}<.05$. For the main effect of concentration, mean wheel-running rates for the $0 \%, 2.5 \%, 5 \%, 10 \%$, and $15 \%$ sucrose conditions were $30.54,33.81$, 35.30, 38.17, and 37.74 revolutions/min, respectively. Bonferroni comparisons showed that wheel-running rate on $0 \%$ sucrose was lower than that at all other concentrations (all ps <.05); on $2.5 \%$ sucrose, wheel running was lower than that at $10 \%$ and $15 \%$ concentrations, both ps $<.005$; on $5 \%$ sucrose, wheel running was lower than that at $10 \%$ concentration, $\mathrm{p}<.05$. With respect to the deprivation by concentration interaction, Bonferonni comparisons showed that under ad-lib feeding, wheel-running rates at $10 \%$ and $15 \%$ sucrose were significantly higher than those at $0 \%, 2.5 \%$, and $5 \%$ sucrose concentrations, all ps $<.05$. By contrast, under deprivation, wheel-running rates at all concentrations were significantly higher than those at $0 \%$ sucrose, all ps $<.05$, and wheel-running rates at $5 \%, 10 \%$, and $15 \%$ were significantly higher than those at $2.5 \%$, all ps $<.05$. This analysis also revealed that at $0 \%$ sucrose, the difference in wheel-running rates between deprived and ad lib conditions approached significance, $\mathrm{p}=.08$.

Figure 2 shows mean latencies to initiate running as a function of feeding condition and concentration for individual rats and the group. Under ad-lib feeding, 
most rats (NQ 3,10,11,12,17, and 19) showed increases in latency to initiate running as concentration increased. Similarly, most food-deprived rats (NQ 1, 2, 4, 9, 18, and 19) showed an increase in latency with concentration. In others (NQ 3 , 10,11 , and 12), latencies increased up to $5 \%$ and then decreased. A three-way repeated measures ANOVA showed only a significant main effect of concentration, $\mathrm{F}(4,36)=22.50, \mathrm{p}<.001, \eta_{\mathrm{p}}{ }^{2}=.73$. Mean latencies to initiate wheel running for the $0 \%, 2.5 \%, 5 \%, 10 \%$, and $15 \%$ sucrose concentrations were $4.09,5.02,6.01,6.41$, and $6.63 \mathrm{~s}$, respectively. Bonferroni comparisons showed that latencies at all concentrations were longer than those at $0 \%$, all ps $<.05$, and latencies at $5 \%, 10 \%$, and $15 \%$ were longer than at $2.5 \%$, all ps $<.05$.

As would be expected, body weight (g) under ad-lib feeding, increased over time with the lowest body weight associated with the first concentration received either $0 \%$ or $15 \%$ depending on order (ascending or descending). For rats on the ascending order, mean weights for the $0 \%, 2.5 \%, 5 \%, 10 \%$, and $15 \%$ concentrations were $284.4,290.4,300.2,302.8$, and $306.6 \mathrm{~g}$, respectively. For the descending order, mean weights for the $15 \%, 10 \%, 5 \%, 2.5 \%$, and $0 \%$ concentrations were 280.5 , 286.6, 291.6, 295.0, and 295.2, respectively. At the end of the ad-lib condition, the mean weight across all rats was $301.44 \mathrm{~g}$ (S.D. $=10.94 \mathrm{~g})$. Under deprivation, body weight was controlled for rats on both orders. Average weights ordered by concentration ( $0 \%$ thru $15 \%$ ) were $258.2,258.9,258.8,260.1$ and $261.2 \mathrm{~g}$ for the ascending group. For the descending group, these values ordered by concentration (15\% thru 0\%) were 259.4, 259.4, 257.9, 260.5, and 259.0 g, respectively. Average body weight under deprivation over all concentrations for all rats was $259.4 \mathrm{~g}$ (S.D. 
$=2.28 \mathrm{~g}$ ). For rats on the ascending order, body weights on the last sucrose concentration (15\%) under deprivation were, on average, $85.28 \%$ of body weights in the $15 \%$ condition under ad-lib feeding. For rats on the descending order, body weights on the last concentration ( $0 \%)$ under deprivation were, on average, $87.76 \%$ of body weights on the last concentration (0\%) under ad-lib feeding. An independent samples t-test showed that there was no significant difference in the levels of deprivation between the two orders, $\mathrm{t}(9)=-1.43, \mathrm{p}=.19$.

\section{Discussion}

In the current study, extrinsic sucrose reinforcement increased wheelrunning rates under both ad-lib and deprived conditions. Under free feeding, only the highest concentrations of sucrose increased wheel-running rates. One implication is that automatically reinforced wheel-running rate is only weakly related to extrinsic reinforcement when rats are not motivated by food deprivation. By contrast, under food deprivation, even the lowest sucrose concentrations $(2.5 \%$ and 5\%) were sufficient to increase operant wheel-running rates. Thus, food-related operant wheel running is highly sensitive to low values of extrinsic sucrose reinforcement.

Consistent with prior findings (Belke, Mann, \& Pierce; Belke \&Pierce, 2015), latency to initiate wheel running following the delivery of solution was shortest at $0 \%$ concentration, as rats did not consume the water and initiated running sooner. Latency increased at the lowest sucrose concentration ( $2.5 \%$ solution), as rats now consumed the solution—although there were considerable individual differences in 
consumption, particularly in the ad-lib condition. Latency further increased at 5\% concentration and then remained relatively unchanged at higher concentrations. The lack of significant differences in latency to initiate wheel running at the $5 \%$ concentration and above would be expected if consumption time did not change with sweetness of the solution.

The interaction of concentration and deprivation on wheel-running rate is central to the current study. Under ad-lib feeding, only the highest concentrations significantly increased wheel-running rates. Deprivation, on the other hand, enhanced sensitivity to sweetness and calories. Concentrations with low efficacy for increasing wheel-running rate under free feeding reinforced wheel-running rate when rats were food deprived. One possibility is that the motivation for wheel running changed with food deprivation (Belke and Pierce, 2016); alternatively, deprivation could have shifted the function relating sucrose concentration to wheelrunning rates.

Under deprivation, wheel-running rates rose quickly at low concentrations and then plateaued. Under ad-lib conditions, rates increased most at high concentrations. This different sensitivity to the amount of sucrose in the solution would account for similar wheel-running rates at $15 \%$ sucrose under ad lib and deprived conditions-resulting from the plateau in wheel-running rates under deprivation and the rise in wheel-running rates with concentration under free feeding. One possibility is that the effect of sucrose concentration on wheel-running rate indicates the value of sucrose under ad-lib and deprived conditions; if so, the two functions (ad lib vs. deprived) should be similar, but shifted with respect to the 
amount of sucrose in the solution. Whether food deprivation shifts the function between sucrose concentration and wheel-running rates, however, would require further investigation over a greater range of concentrations.

Deprivation induced by low body weight typically increases rate of wheel running and also the rate of lever pressing for the opportunity to run (Belke, 1996, 2004). In the current study, an effect of deprivation independent of sucrose should have been observed in the $0 \%$ (water) condition. Wheel-running rates under food deprivation should have been higher than under free feeding; however, this was not the case. The average wheel-running rate was higher when the rats were deprived, but not significantly so. Similar wheel-running rates for ad-lib and deprived groups at $0 \%$ could be due to an order effect in the ad-lib condition, as free feeding rats on the ascending series (beginning at 0\%) would lead to lower body weights than free feeding rats on the descending order (ending with $0 \%$ ).

Results of the present study are consistent with the Premack Principle (Premack, 1959, 1962), assuming that amount of wheel running and rate of wheel activity are related as when a given number of revolutions occurs in a fixed period. Based on automatic reinforcement, wheel running would have a higher free-choice probability than operants such as lever pressing; also research shows that the opportunity for wheel running functions as reinforcement for operant lever pressing (Belke, 1997; Belke \& Pierce, 2014, 2015; Collier \& Hirsch, 1971; Iversen, 1993; Kagan \& Berkun, 1954; Premack, 1962; Premack, Schaeffer, \& Hundt, 1964). To ensure that consuming sucrose functions as reinforcement for wheel running, the probability of consuming sucrose must be greater than for running in a wheel. 
Under ad-lib feeding, the probability of consuming low concentrations of sucrose $(2.5 \%$ and $5 \%)$ would be less than for running in a wheel; thus, these concentrations would not function as reinforcement for operant wheel-running rate. By contrast, consumption of sweeter concentrations such as $10 \%$ and $15 \%$ would have a higher free-choice probability than wheel running and function as reinforcement for operant, wheel-running rate.

Deprivation of food in the deprived condition, on the other hand, would increase the probability that rats would consume the low sucrose concentrations more than at the high concentrations. The increased probability of consumption for low concentrations of sucrose would result in low concentrations functioning as reinforcement for operant wheel-running rate. As noted, food deprivation also increases the probability of wheel running, both amount and rate. But the current findings indicate that the probability of consuming sucrose (as source of sweetness and calories) is higher than wheel running when animals are at $85 \%$ body weight and the food supply is limited, although wheel running would become the higher probability response at more extreme levels of food deprivation (Epling, Pierce \& Stefan, 1983; Pierce, Epling \& Boer, 1986).

Our study raised two fundamental questions. Are the relations between sucrose concentration and operant response rate different for lever pressing and wheel running under ad-lib and deprived conditions? Does automatic reinforcement of wheel running alter the relation between sucrose concentration and operant response rate? Answers to these queries are difficult to find, as few studies have examined the effect of sucrose concentration on lever-pressing rate in 
ad-lib and deprived rats. Leslie (1977), however, investigated the effect of $0 \%, 4 \%$, $8 \%$ and $16 \%$ sucrose solutions on operant lever-pressing rates on a variableinterval (VI) 60 -s schedule by three male hooded rats at ad-lib weight and at $80 \%$ of ad-lib weight. Each concentration remained in effect for five sessions. Under ad-lib feeding, lever-pressing rates increased from $0 \%$ to $4 \%$ but remained relatively unchanged at $8 \%$ and $16 \%$ sucrose. In contrast, under deprivation, lever-pressing rates did not differ from those under ad-lib conditions at $0 \%$ and $2 \%$ sucrose but increased markedly at $4 \%$ and $8 \%$, with no further increase at $16 \%$. Under ad-lib conditions, lever-pressing rates rose slowly toward a rate averaging close to 10 presses/min at the highest concentrations. Under deprived conditions, leverpressing rates beyond $2 \%$ sucrose rose rapidly with average rates of approximately $33 \mathrm{presses} / \mathrm{min}$ at $8 \%$ and $35 \mathrm{presses} / \mathrm{min}$ at $16 \%$ sucrose.

A general comparison of Leslie's (1977) results with those from the current study suggests that both lever-pressing rates and wheel-running rates were more sensitive to changes in sucrose concentration under deprivation than free feeding; however, increases in lever-pressing rates became substantially greater (a 35-fold increase from $0 \%$ to $16 \%$ ) as sucrose concentration increased. Notably, at the highest concentration, lever-pressing rates under deprivation in Leslie's study were much higher than for free-feeding rats. In our study, at the highest concentration (15\%), wheel-running rates under deprived and ad-lib conditions did not differ. The difference in results between Leslie and the current study could have arisen from the nature of the operants, lever pressing and wheel running. Lever pressing has an operant level close to zero, but wheel-running rates shows a moderate 
operant level maintained by automatic reinforcement of the activity itself (Skinner, 1957; Vaughn \& Michael, 1982). Other differences between Leslie’s findings and those of the current study make comparisons difficult; these include strain and gender of rats, the level of deprivation, the type of schedule, and the number of sessions on each schedule. Future research should provide a direct comparison of the effects of sucrose concentration on operant wheel-running and lever-pressing rates, using the same reinforcement schedule within the same rats under both ad-lib and deprived conditions. Such a study would help to clarify the relation between sucrose concentration and operant responding when the operant generates or does not generate automatic reinforcement.

Two potential limitations of the present study are noteworthy. One is that the body weight in the rats under ad-lib feeding increased and this increase in body weight could have lead to the weaker effect of sucrose on wheel running. Although possible, this is unlikely due to the counterbalancing of concentration orders. Although increases in concentration covaried directly with increases in weight for rats on the ascending order, the opposite occurred for rats on the descending sequence. Averaging across these orders would mitigate the impact of this body weight increase on assessment of the effect of concentration on wheel running. Another potential limitation is the effect of age on wheel running, as younger rats were used in our study. In fact, Holloszy (1997) showed that wheel running decreased from approximately $6000 \mathrm{~m}$ /day at 5 months to less than $2000 \mathrm{~m} /$ day at 35 months in mildly food-restricted and substantively food-restricted rats. Mild deprivation was used in Holloszy's study as ad-lib fed rats decreased running 
markedly after just a few months. Holloszy's research suggests that rats' wheelrunning rates in the current study would have declined with age over the 9 months (from 3 to 12 months of age) required to complete the experiment. As ad-lib and deprived conditions were not counterbalanced in our study, the decline in wheel running with age potentially confounded the effects of sucrose concentration.

With respect to this possibility, we note that Holloszy (1997) measured wheel running over a 24 -hour period, and it is not clear that wheel-running rates would decline with age within the brief operant sessions arranged in our study. Furthermore, counterbalancing sucrose concentration across ad-lib and food deprived conditions to control for age effects creates a different confound in that adlib feeding following deprivation differs from ad-lib feeding before deprivation, as deprivation alters physiology (Dulloo, 2005). Another way to address the potential confounding by age is to use short exposures to each concentration (Leslie, 1977); however, brief presentation of sucrose concentrations leads to uncertainty about the stability of the dependent measures. In addition, we note that the potential decline in wheel running with age would have occurred in the deprivation condition of our study, but deprivation would have reduced this decline, opposing the age effect. In sum, it is difficult to be sure whether (or how) age of rats played a role in the present findings. Further research is required to assess the potential effects of age on wheel-running rate with brief operant procedures in ad-lib and deprived rats.

Overall, our study indicates that wheel running is an operant that generates automatic reinforcement, which limits the effect of extrinsic reinforcement on this 
behavior. In the current study, we investigated extrinsic reinforcement of wheelrunning rate over a range of sucrose concentrations under ad-lib and deprived feeding conditions. When rats were free feeding, high sucrose concentrations were required to increase wheel-running rate. Food deprived rats, however, increased wheel-running rate across all sucrose concentrations above $0 \%$ (water). Under both deprivation conditions (ad lib and deprived), wheel-running rates were increased equally by the sweetest concentration (15\% sucrose). While operant lever-pressing rate is sensitive to sucrose reinforcement when rats are food deprived, leverpressing rates at high sucrose concentrations for deprived rats exceed those for rats on ad lib feeding. Together these observations indicate that the function relating sucrose concentration to operant responding depends on the nature of the operant behavior, involving responses that generate or do not generate their own automatic reinforcement. 


\section{References}

Bailey, C.S., Hsiao, S., King, J. E. 1986. Hedonic reactivity to sucrose in rats:

Modification by pimozide. Physiology \& Behavior, 38, 447-452,

http://dx.doi.org/10.1016/0031-9384(86)90409-9

Belke, T.W. 1996. The effect of a change in body weight on running and responding reinforced by the opportunity to run. The Psychological Record, 46, 421-433.

Belke, T. W. 1997. Running and responding reinforced by the opportunity to run: Effect of reinforcer duration. Journal of the Experimental Analysis of Behavior, 67, 337-351, http://dx.doi.org/10.1901/jeab.1997.67-337

Belke, T.W. 2004. Responding for sucrose and wheel-running reinforcement: effect of body weight manipulation. Behavioural Processes, 65, 189-199, http://dx.doi.org/ 10.1016/j.beproc.2003.09.006.

Belke, T.W., Hancock, S.D. 2003. Responding for sucrose and wheel-running reinforcement: Effects of sucrose concentration and wheel-running reinforcer duration. Journal of the Experimental Analysis of Behavior, 79, 243-265, http://dx.doi.org/10.1901/jeab.2003.79-243. 
Belke, T.W., Pierce, W.D. 2009. Body weight manipulation, reinforcement value, and choice between sucrose and wheel running: A behavioral economic analysis. Behavioural Processes, 80, 147-156, http://dx.doi.org/10.1016/j.beproc.2008.11.006.

Belke, T. W., Pierce, W. D. 2014. Effect of sucrose availability and pre-running on the intrinsic value of wheel running as an operant and reinforcing consequence. Behavioural Processes, 103, 35-42, http://dx.doi.org/10.1016/j.beproc.2013.11.010

Belke, T.W., Pierce, W.D. 2015. Effect of sucrose availability on wheel-running as an operant and as a reinforcing consequence on a multiple schedule: Additive effects of extrinsic and automatic reinforcement. Behavioural Processes, 116, 1-7, http://dx.doi.org/10.1016/j.beproc.2015.04.011.

Belke, T.W., Mann, S., Pierce, W.D. 2015. Effects of extinction on wheel running and lever pressing as operant behaviors within a multiple schedule of reinforcement. Learning and Motivation, 52, 36-47, http://dx.doi.org/10.1016/j.lmot.2015.09.002.

Belke, T. W., Pierce, W. D. 2016. Wheel-running reinforcement in free-feeding and food-deprived rats. Behavioural Processes, 124, 1-9, http://dx.doi.org/10.1016/j.beproc.2015.11.018 
Collier, G.,Hirsch, E. 1971. Reinforcing properties of spontaneous activity in the rat. Journal of Comparative and Physiological Psychology, 77, 155-160, http://dx.doi.org/10.1037/h0031588

Dulloo, A.G. 2005. A role for suppressed skeletal muscle thermogenesis in pathways from weight fluctuations to the insulin resistance syndrome. Acta Physiologica, 184, 295-307, doi:10.1111/j.1365-201X.2005.01466.x.

Epling, W. F., Pierce, W. D., Stefan, L. 1983. A theory of activity-based anorexia. International Journal of Eating Disorders, 3, 27-46. doi:10.1002/1098108X(198323)3:1<27::AID-EAT2260030104>3.0.CO;2-T

Guttman, N. 1953. Operant conditioning, extinction, and periodic reinforcement in relation to concentration of sucrose used as reinforcing agent. Journal of Experimental Psychology, 46, 213-224, http://dx.doi.org/10.1037/h0061893

Holloszy, J.0. 1997. Mortality rate and longevity of food-restricted exercising male rats: a reevaluation. Journal of Applied Physiology, 82, 399-403.

Iso, H. 1996. Operant conditioning of wheel-running behaviour in rats. The Japanese Journal of Animal Psychology, 46, 41-60, http://dx.doi.org/10.2502/janip.46.41 
Iversen, I.H. 1993. Techniques for establishing schedules with wheel running as reinforcement in rats. Journal of the Experimental Analysis of Behavior, 60, 219-238, http://dx.doi.org/10.1901/jeab.1993.60-219

Kagan, J., Berkun, M. 1954. The reward value of running activity. Journal of Comparative and Physiological Psychology, 47, 108, http://dx.doi.org/10.1037/h0058877

Leslie, J.C. 1977. Effects of food deprivation and reinforcement magnitude on conditioned suppression. Journal of the Experimental Analysis of Behavior, 28, 107115, http://dx.doi.org/10.1901/jeab.1977.28-107

Pierce, W. D., Epling, W. F., Boer, D. P. 1986. Deprivation and satiation: The interrelations between food and wheel running. Journal of the Experimental Analysis of Behavior, 46, 199-210. doi: 10.1901/jeab.1986.46-199.

Premack, D. 1959. Toward empirical behavioral laws: I. Positive reinforcement. Psychological Review, 66, 219-233, http://dx.doi.org/10.1037/h0040891

Premack, D. 1962. Reversibility of the reinforcement relation. Science, 136, 255-257, http://dx.doi.org/10.1007/s10803-012-1571-3 
Premack, D., Schaeffer, R. W., Hundt, A. 1964. Reinforcement of drinking by running: Effect of fixed ratio and reinforcement time. Journal of the Experimental Analysis of Behavior, 7, 91-96, http://dx.doi.org/10.1901/jeab.1964.7-91

Skinner, B.F. 1957. Verbal behavior. Englewood Cliffs, NJ: Prentice-Hall.

Skinner, B. F., Morse, W. H. 1958. Fixed-interval reinforcement of running in a wheel. Journal of the Experimental Analysis of Behaviour, 1, 371-379, http://dx.doi.org/10.1901/jeab.1958.1-371

Vaughn, M.E., Michael, J.L. 1982. Automatic reinforcement: An important but ignored concept. Behaviorism, 10, 217-227.

Vigorito, M., Kruse, C. B., Carretta, J. C. 1994. Differential sensitivity of operant behaviors to changes in the concentration of a sucrose reinforcer: Effects of Pimozide. Pharmacology Biochemistry and Behavior, 47, 515-522, http://dx.doi.org/10.1016/0091-3057(94)90153-8 


\section{Acknowledgement}

The experiment in this report is based on an undergraduate thesis submitted by the third author in partial fulfillment of a B.Sc. degree at Mount Allison University, Sackville, Canada. This research was supported by Grant 0GP0170022 from the Natural Sciences and Engineering Research Council of Canada to the first author. Correspondence regarding this article should be sent to Terry W. Belke, Department of Psychology, Mount Allison University, Sackville, New Brunswick, Canada, E4L 1C7 or via E-mail to TBELKE@MTA.CA. 


\section{Figure Captions}

Figure 1. Mean wheel-running rates (revolutions/min) as a function of sucrose concentration under ad-lib fed and food deprived conditions for individual rats and the group. Standard error bars are plotted for each mean.

Figure 2. Mean latency to initiate running (s) as a function of sucrose concentration under ad-lib fed and food deprived conditions for individual rats and the group. Standard error bars are plotted for each mean. 
REINFORCEMENT OF OPERANT WHEEL RUNNING 28

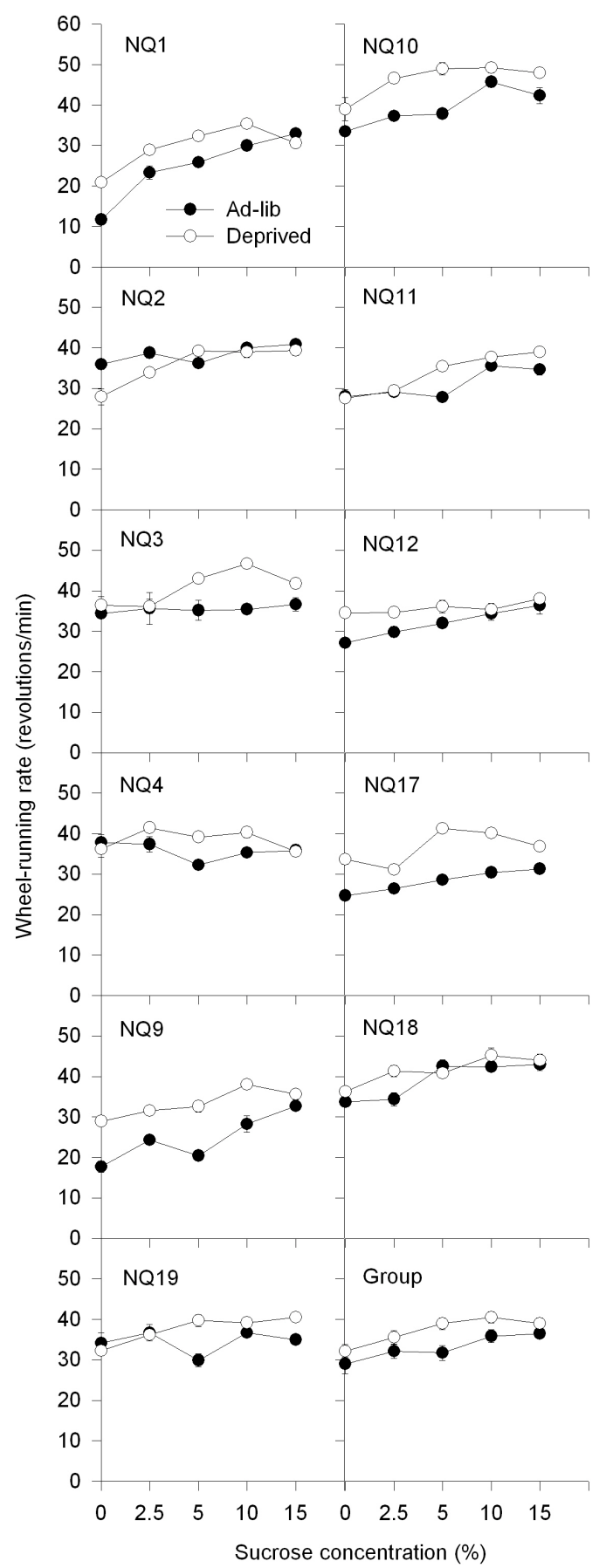




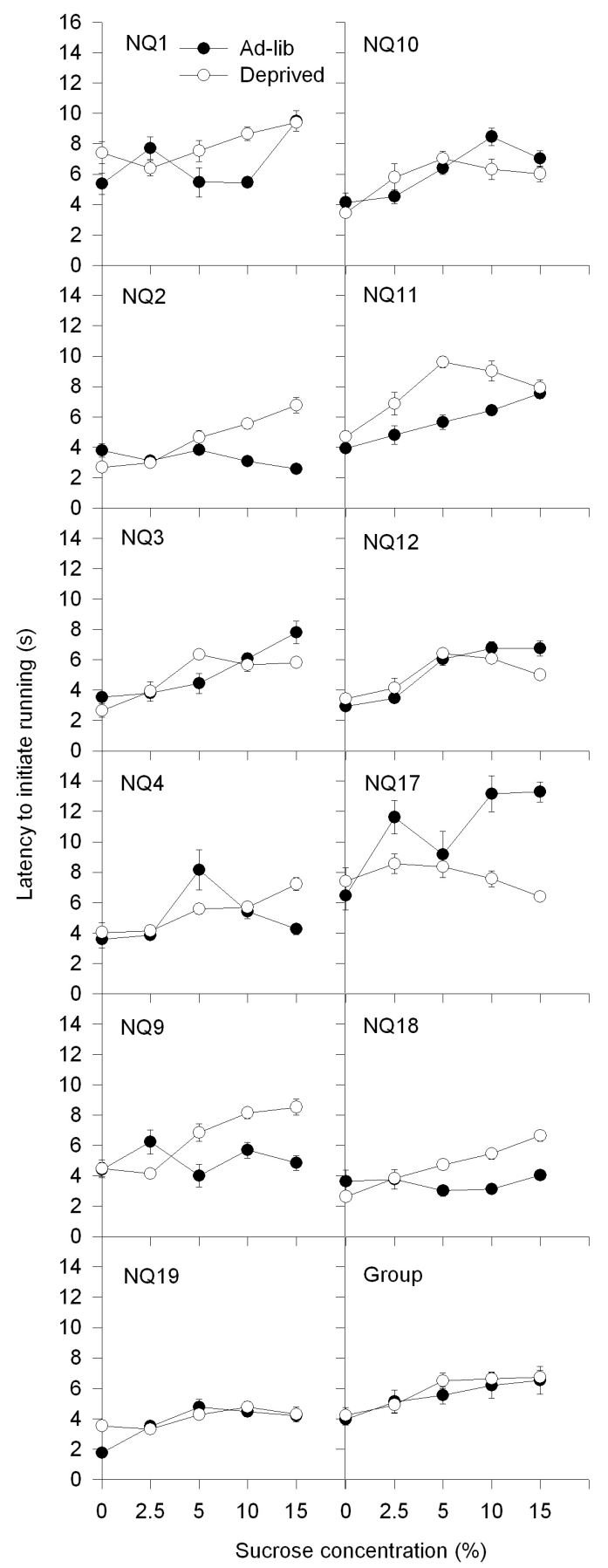

